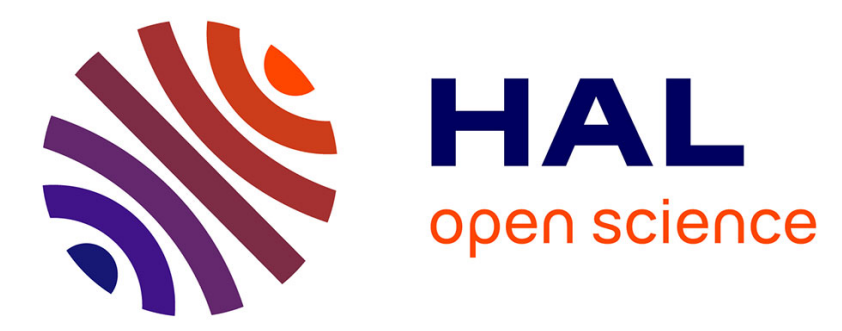

\title{
Deux squelettes humains au milieu de foyers de l'époque Moustérienne
}

\author{
Denis Peyrony, Louis Capitan
}

\section{To cite this version:}

Denis Peyrony, Louis Capitan. Deux squelettes humains au milieu de foyers de l'époque Moustérienne. Revue de l'Ecole d'Anthropologie de Paris, 1909, 12, pp.403-409. halshs-01076288

\section{HAL Id: halshs-01076288 \\ https://shs.hal.science/halshs-01076288}

Submitted on 21 Oct 2014

HAL is a multi-disciplinary open access archive for the deposit and dissemination of scientific research documents, whether they are published or not. The documents may come from teaching and research institutions in France or abroad, or from public or private research centers.
L'archive ouverte pluridisciplinaire $\mathbf{H A L}$, est destinée au dépôt et à la diffusion de documents scientifiques de niveau recherche, publiés ou non, émanant des établissements d'enseignement et de recherche français ou étrangers, des laboratoires publics ou privés.

\section{(1) $\$(0)$}

Distributed under a Creative Commons Attribution - NonCommercial - ShareAlikel 4.0 


\section{REVUE}

$\mathrm{DE}$

\section{L'ÉCOLE D'ANTHROPOLOGIE}

\section{DE PARIS}

REGUEIL MENSUEL

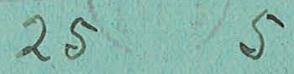

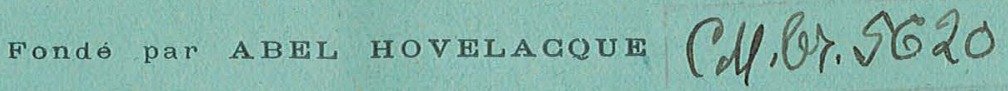

Publié par les Professeurs

DIX-NEUVIÈME ANNÉE. - XII. - DÉGEMBRE 1909

\section{EXTRAIT}

DEUX SQUELETTES HUMAINS

AU MILIEU DE FOYERS DE L'ÉPOQUE MOUSTÉRIENNE

Par MIM. GAPITAN et PEYRONY

\section{Millan \\ Brun tiss:Cn-Malan Bun \\ Anne-Lise, $0=U M R$ \\ Anne- \\ 7194, \\ FÉLIX ALGAN, ÉDITEUR \\ ou=Bibliotheque, email=biph@mnhn.fr 108, BOULEVARD SAINT-GERMAIN, 108 \\ PARIS, $6^{\circ}$}

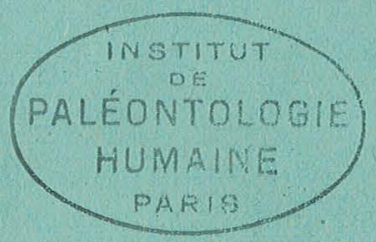


CAPITAN et PEYRONY. - DEUX SQUELETTES HIIMAINS

DEUX SOUELETTES HUMAINS

AU MILIEU DE FOYERS DE L'ÉPOQUE MOUSTÉRIENNE

Par MM. le Dr CAPITAN et PEYRONY

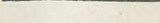

Nous avons l'honneur de communiquer à l'Académie 1 les résultats encore inédits (car nous avous voulu en réserver la primeur à l'Académie) de nos déconvertes toutes récentes dans le département de la Dordogne de restes humains reposant dans des foyers indiscutablement de l'époque mousté rienne, c'est-à-dire correspondant à la base du quaternaire moyen.

La première découverte a été faite par l'un de nous (Peyrony) à 5 kilomètres de Sarlat, au lieu dit le Pech de l'Azé, dans une petite grotte creusée au milieu d'une falaise abrupte, appartenant à la Cie d'Orléans qui a autorisé

Il existe dans cetle grotte une couche archéologique fouillée jadis non scientifiquemente devant la grotte. Là, elle mesure 1 mètre environ d'ép terrasse qui existe devant verte par 3 mètres de gros blocs calcaires et d'éboul is provenant de l'effondrement du plaíond qui se trouvait devant la grotte comme une sorte d'auvent formant abri.

Lorsque nous eûmes enleré complètement l'éboulis, lo partie supérieure de la couche archéolo enture de profondeur, dans l'intérieur d'un enfant, âgé de cinq à six ans et écrasé Tout autour de lui, se trouvaient en abondance des ossements brisés artificiellement et des dents de bovidés, de cerfs, de chevaux, de caprins et de rennes, puis des silex nombreux : pointes et couteaux-racloirs bien retouchés sur une face, du type mousterien supérieur. Au-dessous du cràne, la couche moustérienne renfermait de belles haches du type de St-Acheul.

1909

A. Communication faite à l'Académie des Inscriptions, séance du 19 novembre Les photographies accompagnant la présente note ont été exécutées par H. Lucas sous notre direction. Nos clichés photographiques ont été reproduits voulu mettre à notre disposition ces remarquables clichés typographiques que nous reproduisons. Ce dont nous remercions vivement son directeur, M. Baschet, et le rédacteur de l'article accompagnant les figures, Mi. F. Honoré.
Le reste du squelette manquait, soit qu'il ait été enlevé dans les fouille antérieures faites en avant de l'éboulis, soit qu'il ait été seul dans les débris accumulés par les moustériens.

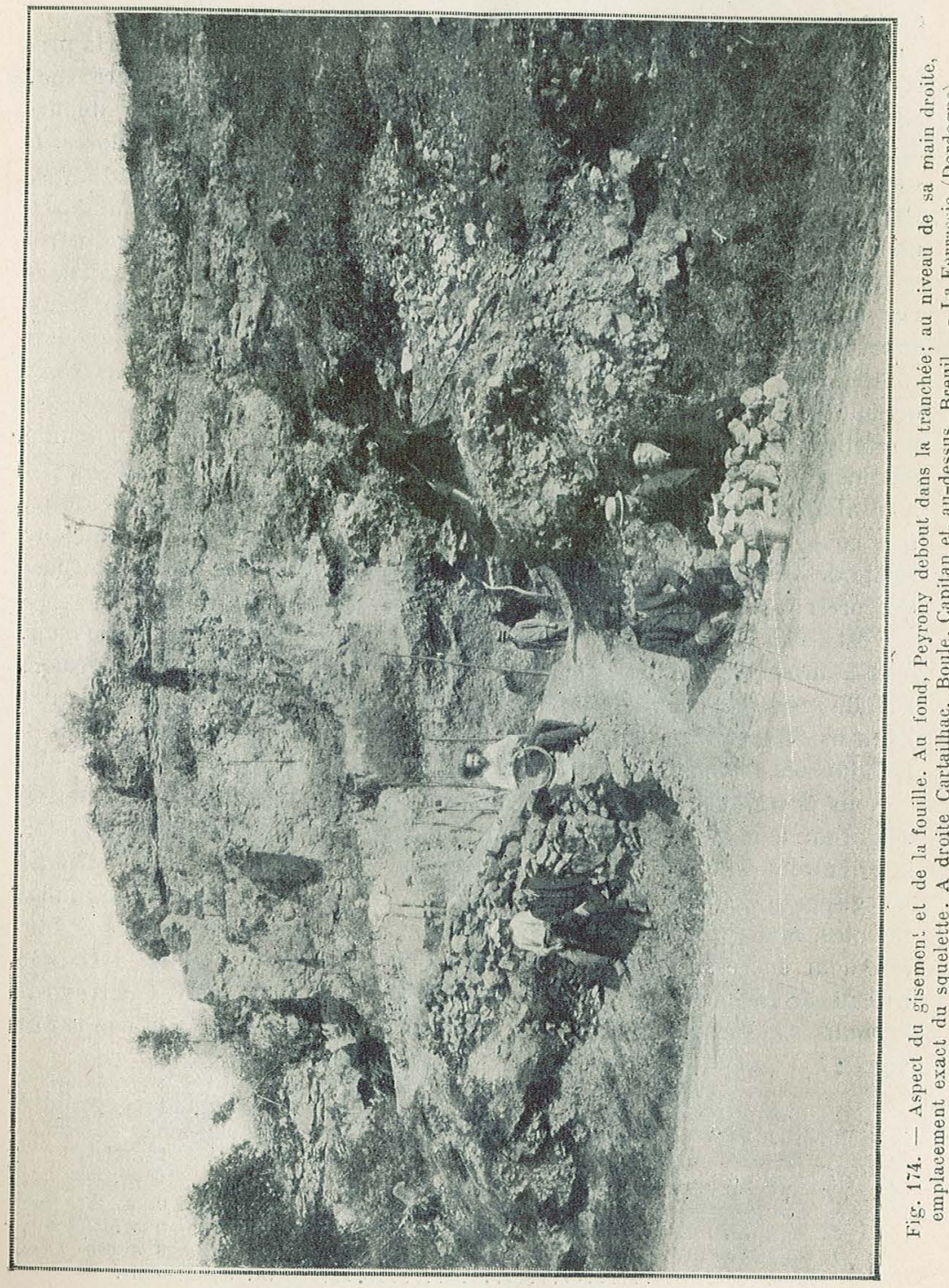

S'agissait-il d'un rite funéraire consistant en l'enfonissement sous un foyer du cadavre de l'enfant; ou bien ce cràne d'enfant aurait-il été aban- 
donné dans ce foyer par les moustériens, au même titre que les débris seux brisés pour en extraire la moelle des animaux ayant servi à leu alimentation? ou entin le cadavre aurait-il été dévoré par les hyènes et n'en serait-il resté que ce cràne brise? Il est bien dithcile de se prond

La seconde découverte a été faite à 32 kilomètres à vol d'oiseau de la préen dans le trés important giserassie, près du Bugue (Dordogne), que l'un de nous (Peyrony) explore depui dix ans et sur lequel nous avons ensenble publie deja plasious notes. s'agit là d'un abri considérable qui a éte habité pendant fort longtemps, chaque population correspondant à une époque determine, aya induse une couche de débris provenant de son alimentation et de son industrie spéciale. Quatre couches (qui sont de bas en haut : acheuléenne, moustérienne, aurignacienne inferrieure et aurignaciente moyente) se sont ain superposées, differentes par leur couleur, lindustrie et la taune qu'sles contiennent. Après le dépôt de la quatrième, le plafond de l'abri s'est écroulé et c'est entre et sur les blocs et les pierres de cet éboulis que de nouvelles populations (aurigaciennes supérieures) ont abandoné une cinquième couche de débris de leur industrie et de la fanne ayant servi a leur alimentation ${ }^{1}$. Au-dessus il s'est deposé de lhumus et, par-dessus, une couche de pierrailles et ainsi s'est formé un depot d'une épaisseur totale de $3 \mathrm{~m} .90$. On peut se rendre compte sur la figure 175 de l'aspect que présentait la coupe de ce gisement avant notre découverte.

ar en bas), c'est-à-dire entre l'acheuléen et le moustérien, que, le 17 septembre dernier, I'un de nous (Peyrony), accompagné de M. Raveau (de Bordeaux) aperçut deux os faisant légèrement saillie hors de la coupe. Ils les dégagèrent et reconnurent facilement un tibia et un fémur humains.

Nous prévinmes nos amis les professeurs Boule, Cartalhac, Breuil, qui se rendirent à notre appel. MM. Féaux, Bouyssonie, Bardon, Raveau, etc., se joignirent à eux. Le 27 septembre, après étude soigneuse des conditions du gisement, après avoir constaté que les couches etaient absolument intactes, nous procédâmes en leur presence et avec leur concours a l'enlèrement successif par tranches horizontales - correspondant à chaque niveau archéologique - et ce sur une surface de 4 mètres carrés, de l'éboulis supérieur. puis des couches archéologiques, suivant la coupe que vici

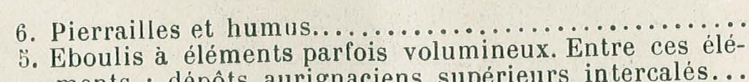
ments : dépôts aurignaciens supérieurs intercalés.. 1 m. 20 Aurignacien moyen. Aurignacien inférieur............................ $00 \mathrm{~m} .20$

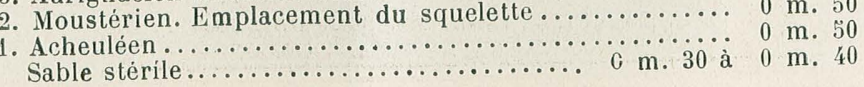
1. Il n'y a pas à établir ici l'exactitud

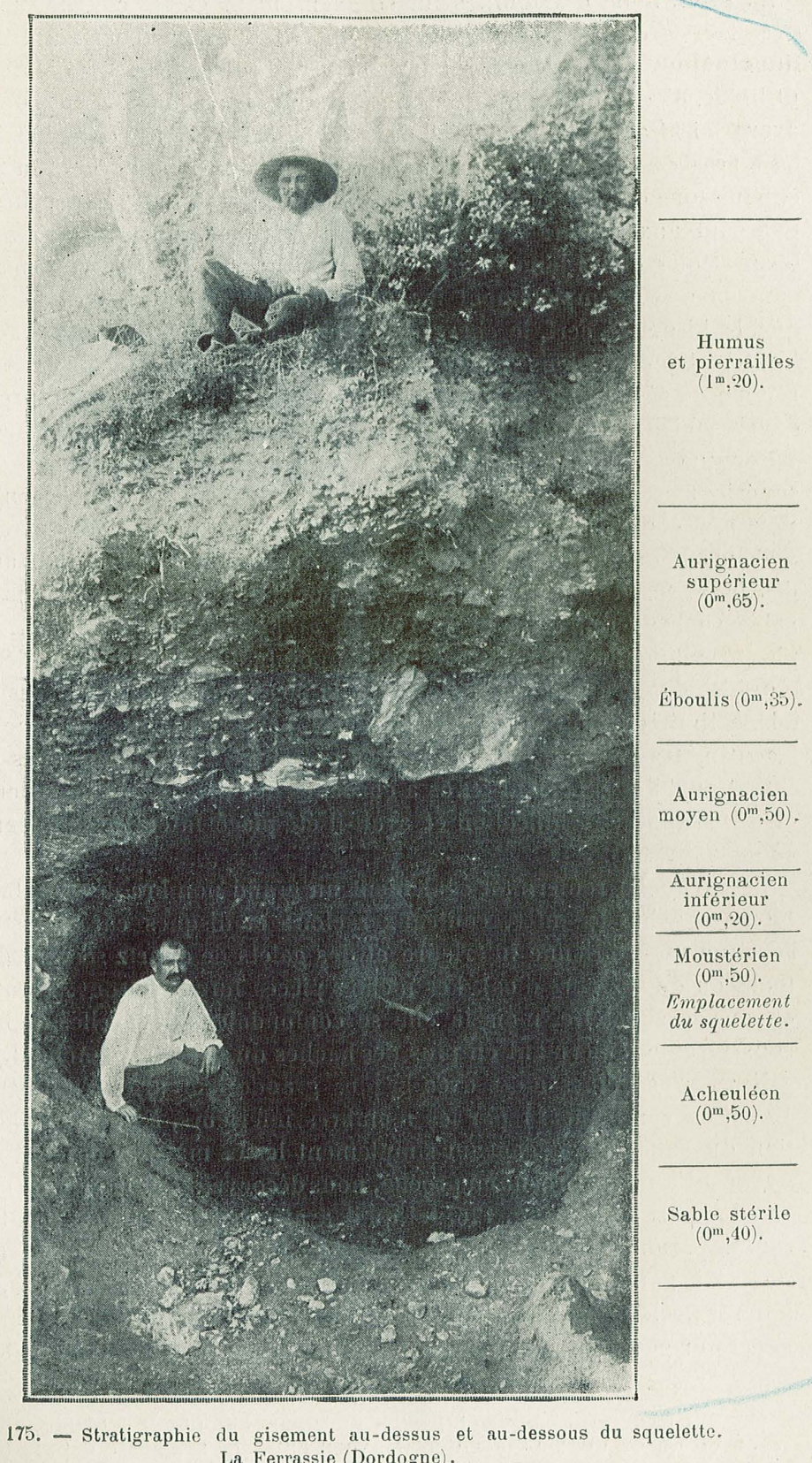

Fig. 175. - Stratigraphie du gisement au-dessus et au-dessous du squelett
La Ferrassie (Dordogne). 


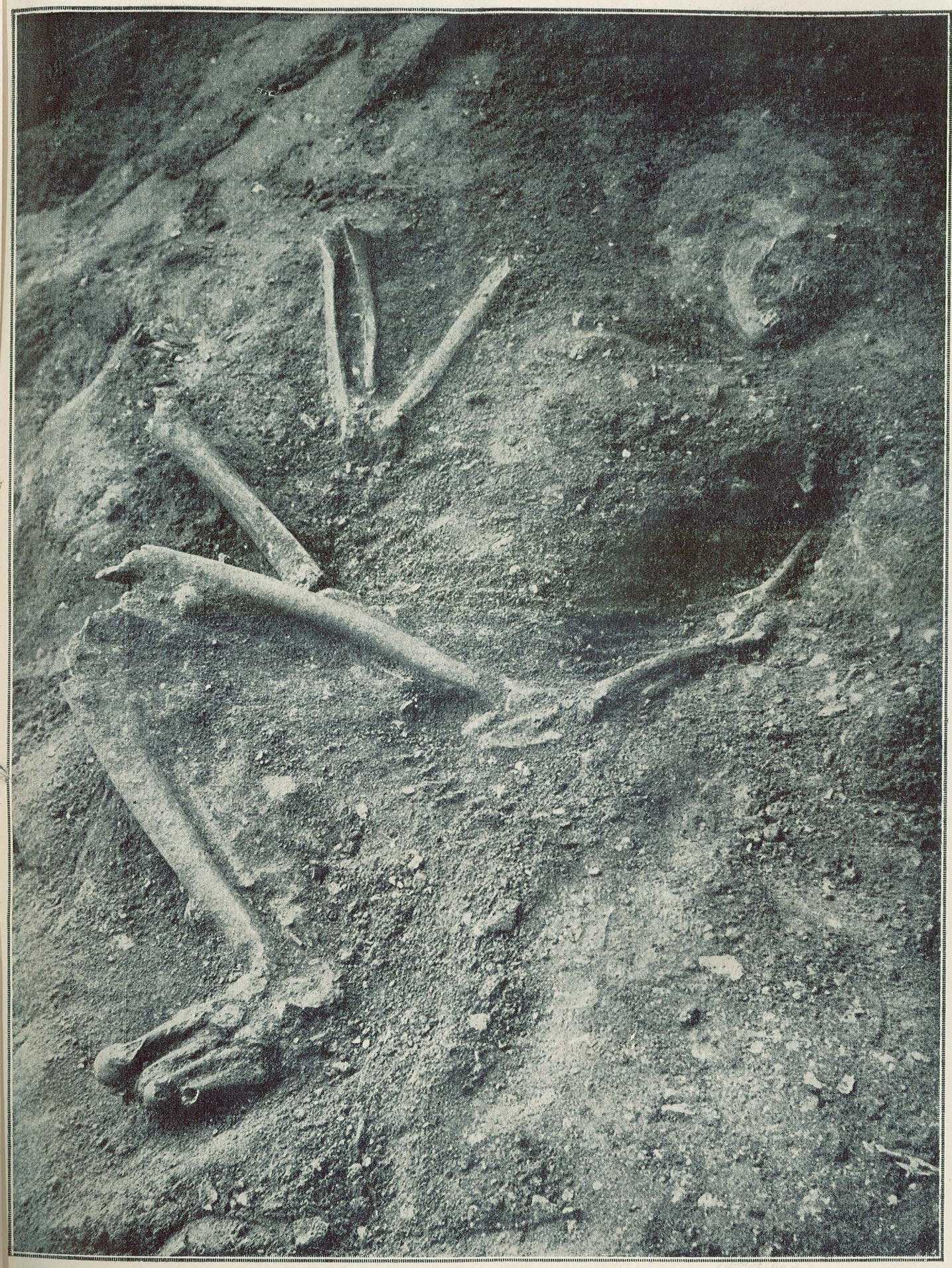

nt classé couche par couch très faciles d'ailleurs à reconnaître du fait de leur coloration différente Nous pûmes ainsi recueillir un grand nombre de silex taillés, de pierres utilisées, d'os et de dents des animaux mangés par chacune des populations ayant laissé ainsi, - englobées dans du sable, de la terre, des cendres, les traces de leur habitat prolongé en ce point. L'industrie et la faune de chaque zone ont des caractères différents de ceux des autres couches. Ainsi aux couteaux-racloirs et lames epaisses du monstérien succèdent les burins, les grattoirs et les lames minces bien retouchées, les lames à encoches de l'aurignacien, complètement inconnus des moustériens. De même, tandis que le bison, le cerf, le cheval (caractérisant un régime de prairies et de forêts) abondent dans le moustérien et que le renne (caractérisant un régime de plaines glacées) y est rare, celui-ci devient plus nombreux dans l'aurignacien, alors que les autres animaux y diminuent beaucoup. Lorsque nous eùmes ainsi enlevé toutes les couches, depuis l'éboulis supérieur jusqu'à la couche 2 (moustérienne), et celle-ci presque en totalité, nous apercûmes trois pierres plates de $0 \mathrm{~m} .20$ de côté environ et placées l'une à l'endroit où se trouvait le crâne et les deux autres à peu près au niveau des bras. Sur toute la surface correspondant au squelette, dans la terre sableuse brun rougeàtre de la couche archéologique, il existait, en bien plus grand nombre que dans les autres points du même niveau, de grande esquilles d'os d'animaux, portant sur la face extérieure des traces de martelage limitées en un, parfois en deux points, sur une surface de 2 à 3 centimètres carrés ${ }^{1}$. L'n de ces os présente une série de fines entailles intentionnelles rappelant les os à encoches des niveaux aurignaciens. Peutêtre avait-il une signification et avait-il été placé intentionnellement à côtè du squelette.

Cette couche renfermait également un grand nombre de silex taillés fort bien retouchés (nointes, couteaux-racloirs et disques caractéristiques de l'époque moustérienne inférieure et des galets de quartz servant de percuteurs ou de casseurs d'os). Les mêmes pièces furent retrouvées autour, sur et sous le squelette, mais là elle se confondaient avec celles de la couche sous-jacente renfermant en plus des haches du type de St-Acheul.

Nous procédâmes alors avec la plus grande lenteur et d'infinies précautions, en commencant par les membres inférieurs, à une véritable dissection du squelette, dégageant simplement les os mais les laissant en place. Avancant ainsi de proche en proche, nous découvrîmes le squelette entier'qu nous appart a contemporains, les moustériens, éendu sur le dos, le tronc légèrement incliné à i demi fléchis supérieur gauche le long du corps, la main gauche au niveau de la hanche

1. Nous ne pouvons nous empêcher de songer aux galets martelés de la mème la présence dans un très grand nombre de sépultures bretonnes mégalithiques. 
gauche, le bras droit plié et la main droite à peu près au niveau de l'épaule, la tête tournée à gauche, la mâchoire largement ouverte.

Tous les os du squelette, parfois brisés par le poids énorme des terres qui le recouvraient, étaient en place, dans leurs connexions anatomiques encore solides Les os du pied et de la main droits seuls avaient ite déplacés, probablement par un petit oarnassier ou un rongeur, et ont partie disparu.

Toutes les constatations ayant été minutieusement faites, les photographies prises, nous enlevines ave le plus gratobres. Puis, ayant creusé 'une rainure profonde tout autour du bes memrecourrimes les os de papior d'étain puis d'une épa En creusant alors En crenant alors lentement audessour

La même opération nous permit d'extraire ce qui restait du thorax, puis, en troisième lieu, - et ce avec un soin tout spécial, - le crâne et un large - d'une forte enveloppe de plâtre.

Ces diverses parties pourront donc être remontées sans qu'il y en ait une sedle parcelle égarée, puisque toute la terre les enveloppant restera la conaration dans le laboratoire. C'es grstes, istes, est employée pour la récolte d ossements humains fossiles. C'est tion phié au Nous a Nous avons, avec nos savants amis, longuement discute, sur place, les conditions qui avaient dú présider au dépôt de ce corps sur ce point au l'idé d' lepoque mousterientice. Nous avons eté unanimes pour repousse Riée d une cen accidentelle en ce lieu, le cadavre étant resté sur place. then dans le terrain n'indique un effondrementa ce moment; puis il y avait à un grand abri très habite (les débris archéologiques lindiquent); le sujet n'a donc pu ètre isolé en ce point. D'ailleurs il eùt été rapidement et infailliblement dévoré par les hyènes.

Nous avons pensé qu'il avait dù y avoir là un rite funéraire. Le cadavre aurait été place dans un coin de l'abri, sur une partie du sol légèremen déclive vers la paroi, et près de cette paroi. Le sol etait formé par les débris usagés et les foyers des habitants antérieurs, les acheuléens. (Il avait une coloration jaune, tandis que celui qui entourait le squelelte est brun rougeâtre.)

Le cadavre avait eu les jambes fortement ployées, suivant le même rite probablement que les squelettes négroïdes des couches inférieures de la grotte des Enfants de Menton. Les trois pierres dont nous avons parlé ci-dessus avaient peut-être été placées intentionnellement, l'une sur la tête et les deux autres sur les épaules. Peut-être aussi, les os d'animaux martelés avaient-ils été volontairement étendus sur lui, conformément à un rite spécial. Il avail ensuite été recouvert, soit de branchages, soit de peaux, peut-être d'un peu de terre et de débris formant le sol. Mais il n'avait certainement pas éte entere dans une losse.

Protégé par le voisinage des moustériens habitant l'abri, il ne fut pas dérange, saul par de petits carnassiers ou des rongeurs qui dispersèrent les os du pied et de la main droits. Peu à peu les débris usagés des habitants de labri (terre, cendres, éclats d'os et de silex, galets) s'étendirent sur lui comme dans le reste de l'abri et furent vraisemblablement étalés et foulés par le va-et-vient des habitants de la grotte. Ainsi se constitua audessus de lui une stratification parfaitement régulière de dépôts archéolo. giques, tout comme dans le reste de l'abri, et se succédant d'àge en àge suivant T'évolution humaine en ce lieu. Sous ce linceul pesant et immuable ce squelette se conserva. La chute du plafond de l'abri, les dépôts meu bles successifs finirent cette œuvre de conservation qui nous a permis de retrouver notre si prodigieusement vieil ancètre, exactement dans la position où ses contemporains l'avaient mis il y a tant et tant de siècles. Nous avons tenu essentiellement à donner à l'Académie des Inscriptions la primeur du récit de notre découverte et des photographies documentaires qui laccompagnent. Nous ferons de même pour l'étude des squelettes et des industries concomitantes, études auxquelles nous nous livrerons des que le delicat et long travail de réparation et de montage des pièces sera terminé. 